\begin{tabular}{|c|c|c|}
\hline & Int.J.Curr.Microbiol.App.Sci (2016) 5(3): 502-512 & \\
\hline & International Journal of Current Microbiology and Applied Sciences & 2. \\
\hline & ISSN: 2319-7706 Volume 5 Number 3(2016) pp. 502-512 & \\
\hline $\begin{array}{l}\text { EXCELLENT } \\
\text { PUBLISHERS }\end{array}$ & Journal homepage: $\underline{\text { http://www.1jcmas.com }}$ & \\
\hline PUBLISHERS & & wirwijijemas.com \\
\hline
\end{tabular}

Original Research Article

http://dx.doi.org/10.20546/ijcmas.2016.503.059

\title{
Role of Acinetobacter as Pathogen of Health Care Associated Pneumonia and Blood Stream Infections in ICU Setting in a Tertiary Care Hospital in New Delhi
}

\author{
Ruchi Girotra*, Raman Sardana, Reetika Dawar, Leena Mehndiratta, Sudha Kansal, \\ Rajesh Chawla, Ruby Naz and Narendra Agarwal
}

\author{
Department of Microbiology, Indraprastha Apollo Hospitals, New Delhi, India \\ *Corresponding author e mail:
}

\begin{abstract}
A B S T R A C T
Keywords

Acinetobacter,

Healthcare

Associated

Pneumonia,

Blood stream

infections, Risk

factors, Intensive

Care Units.

Article Info

Accepted:

20 February 2016

Available Online:

10, March 2016

Invasive diseases due to Acinetobacter is an increasing problem in health care settings and especially in Intensive Care Units (ICU) worldwide. These organisms occur as components of commensal flora of man and animals and are therefore regular contaminants of hospital environment. Purpose-This prospective study was conducted at Indraprastha Apollo Hospitals, New Delhi in the Department of Microbiology and Respiratory and Critical Care Medicine to ascertain the role of Acinetobacter as a Pathogen in Healthcare Associated Pneumonia (HCAP) and Blood stream Infections (BSI). Findings-One hundred and eight consecutive, non repeat isolates of Acinetobacter species isolated from blood and respiratory tract were studied. Nineteen cases were identified together as Healthcare associated pneumonia (HCAP) and blood stream infections (BSI). By applying Fishers Exact Test,the colonization rate vs the infection due to Acinetobacter species rate was calculated to be $82.41 \%$ (BSI+HCAP) and $17.59 \%$ which was found to be significantly in in favour of colonization.(p- value0.001). Tracheostomy was a significant risk factor associated with the development of HCAP due to Acinetobacter species (p-value< 0.05)(Fishers Exact Test) while Immunosuppression ( $\mathrm{p}$-value $\leq 0.05$.) and presence of multiple catheters $(\mathrm{p}<0.05)$ were independent risk factors in BSI infections. Immunosuppression was found to be significantly higher in the BSI group compared to the Non-BSI group. Conclusion- This study shows that mere colonisation by Acinetobacter species should not be considered as an indication for antimicrobial treatment for the same as a very small proportion of Acinetobacter species was responsible for infection.
\end{abstract}

\section{Introduction}

Patients in Intensive Care Unit are at risk of dying not only from their critical illness but also from secondary processes such as healthcare associated infections (Koenig et al., 2006).

Invasive diseases due to Acinetobacter is an increasing problem in health care settings and especially in Intensive Care Units (ICU) worldwide. These organisms occur as components of commensal flora of man and animals and are therefore regular contaminants of hospital environment.

Hence they are increasingly recognised as opportunist pathogens causing serious antibiotic resistant infections ranging from bronchopneumonia to septicemia in compromise patients (Collee et al., 2006). 
It is very difficult to explain the role of Acinetobacter acquisition in the ICU, since Acinetobacter does not always act as an infecting pathogen as it is widely distributed and has tremendous colonising capability.In addition, risk factors for Acinetobacter acquisition may vary in different set ups.

Why Is A. baumannii a Persistent Hospital Pathogen? There are three major factors possibly contributing to the persistence of $A$. baumannii in the hospital environment, i.e.,

Resistance to major antimicrobial drugs

Resistance to desiccation

Resistance to disinfectants.

Resistance to antibiotics may provide certain A. baumannii strains with a selective advantage in an environment, such as the modern ICU, where microorganisms are confronted with extensive exposure to antimicrobials. Several researchers have observed that resistance rates in epidemic $A$. baumannii strains are significantly higher than those in sporadic A. baumannii strains (Dijkshoorn et al., 1996; Heinemann et al., 2000; Jawad et al., 1998; Koeleman et al., 2001).

Though majority of Acinetobacter species isolated tend to be either colonisers or contaminants, they are always thought to be clinically significant by the treating team. Clinical and microbiological studies have to be carried out to define and distinguish Acinetobacter thus isolated to delineate colonisers or contaminants those that have infection.

Although various factors predisposing to Acinetobacter infections have been analysed in different studies, there are only few reports all over the world that have attempted to determine the risks for Acinetobacter infection in Ventilator associated pneumonia and Blood stream related infections. This study was done with objective to isolate Acinetobacter species from clinical specimens of Blood and respiratory species and to further possibly characterise the isolates on the basis of risk factors, clinical correlation etc.

\section{Methodology}

This prospective study was conducted at Indraprastha Apollo Hospitals, New Delhi in the Department of Microbiology and Respiratory and Critical Care Medicine. laboratory in our One hundred and eight consecutive, non repeat isolates of Acinetobacter species isolated from blood (22) and respiratory tract specimens (86) of patients admitted in ICU were studied, with an objective to isolate Acinetobacter species from clinical samples and to further characterise the isolates on the basis of risk factors, clinical correlation and microbiological techniques into HCAP and BSI. All the patients fulfilling the inclusion criteria were enrolled for the study (Table $1)$.

\section{Inclusion Criteria}

All critically ill patients, admitted in Intensive Care Units (ICU), who were suspected for Blood stream infections and Health- care associated pneumonia were considered in this study. Further isolates of Acinetobacter species recovered from blood specimens or respiratory specimens, after 48-72 hours of admission to ICU of such suspected cases were followed up.

\section{Exclusion Criteria}

Repeat samples from the same patient were not considered in the study. 
Any patient with Acinetobacter species being isolated also from sites other than blood and respiratory tract and not fitting into criteria of BSI or HCAP were not considered in the study.

\section{Risk Factors}

Among the risk factors considered for HCAP were hypertension (H),41 (47.67\%), chronic obstructive pulmonary, disease (COPD), 16, (18.60\%), coronary arterial disease (CAD) 16, (18.60)\%, chronic renal failure (CRF), 12, (13.95\%), cerebrovascular accident (CVA) 8(9.30\%), diabetes (D) 22, (25.58\%), immuno-suppression (I) 23, (26.74\%), chronic lung disease (CLD) $1,(1.16 \%)$ and chronic liver disease (CLiD), 5, (5.81\%). Hypertension though generally not a risk factor was a significant clinical finding in the cases taken and therefore taken into consideration.

Steroids 5 (5.80\%), immunosuppressives 2, $(2.32 \%)$, use of previous antibiotics 65 $(75.58 \%)$ was also taken as a risk factor.

Among the risk factors considered For Blood stream infections (BSI) were hypertension, 5 (22.70\%), chronic obstructive pulmonary, disease (COPD), 0 , $(0 \%$,$) coronary arterial disease (\mathrm{CAD}) 2$, (9.09\%), chronic renal failure (CRF), $2,(9.09 \%)$, cerebrovascular accident(CVA) 2(9.09\%), diabetes(D) 7,(31.81\%), immunosuppression (I )2, 9.09\%,chronic lung disease $0,0 \%$ and chronic liver disease,2,(9.09\%).\%). Hypertension though generally not a risk factor was a significant clinical finding in the cases taken and therefore taken into consideration.

Steroids $0(0 \%)$, immunosuppressives 1, (4.54 \%), use of previous antibiotics 21 $(95.45) \%$ was also taken as a risk factor.

\section{Specimen Collection}

Respiratory specimens - Respiratory samples \{sputum, endotracheal aspirate, bronchoalveolar lavage (BAL), transtracheal specimen, tracheostomy secretions etc. were collected aseptically taking all precautions.

For the respiratory specimens, Gram staining was done immediately followed by culture of the specimen after receiving in the Microbiology Laboratory. Semi Quantitative culture was done for BAL, ET and tracheal secretion secretions.

The plates were then incubated at $37^{\circ} \mathrm{C}$ for 18-24 hrs.

\section{Blood for Blood Cultures}

For collecting blood universal precautions were followed. The blood was then withdrawn and 8-10 $\mathrm{ml}$ of blood was injected into appropriate BD BACTEC Plus bottles preferably at bedside., 8,9 For paediatrics purpose $0.5 \mathrm{ml}$ to $5 \mathrm{ml}$ was inoculated in BACTEC PEDS/F culture vials.

Blood culture was carried out in continuous monitoring Automated blood culture system (BACTEC-9120).

The culture plates of positive specimens were incubated at $37^{\circ} \mathrm{C}$ for $18-24$ hours. Growth of organism and colony characters were noted after incubation.

For further processing and identification and susceptibility testing of the organism, Vitek2 compact system was used .

All the patients with blood and respiratory isolates being identified as Acinetobacter 
species were followed up further for clinical correlation .

\section{Clinical Correlation}

The presence of Health-care associated pneumonia (HCAP) were observed according to CDC surveillance definition of Healthcare associated Pneumonia i.e. identified by using a combination of imaging, clinical and laboratory criteria (Pneumonia (Ventilator-associated [VAP] and non-ventilator-associated Pneumonia [PNEU]) Event.).

The patients whose blood stream isolates showed Acinetobacter were observed for presence of any central lines (subclavian, jugular, peripheral inserted central catheter) and the development of BSI.

The correlation in our study was done using the Centers for disease control and prevention (CDC) criteria for Bloodstream Infection Event (Central Line-Associated Bloodstream Infection and Non-central lineassociated Bloodstream Infection) i.e. Patient has a recognised pathogen cultured from one or more blood cultures and organism cultured from blood is not related to an infection at another site.

Patient has at least one of the following signs or symptoms: fever (>38.0oC), chills, or hypotension and organism cultured from blood is not related to an infection at another site and the same common commensal (i.e., diphtheroids [Corynebacterium spp. not C. diphtheriae], Bacillus spp. [not $B$. anthracis], Propionibacterium spp., coagulase-negative staphylococci [including S.epidermidis], viridans group streptococci, Aerococcus spp., and Micrococcus spp.) is cultured from two or more blood cultures drawn on separate occasions. ${ }^{10}$

\section{Statistical Analysis}

P-values were calculated applying Fishers Exact Test.

The following were statistically analysed-

Infection vs colonisation rates due to Acinetobacter species in blood stream infection groups and health care associated pneumonia group,

Co-morbid factors (gender, age, risk factors etc) affecting the outcome (HCAP and BSI), and catheter characteristics in blood stream infections.

Average length of ICU stay.

Tests used were Fisher's Exact Test, Student T -test, Binomial test. (where ever appropriate).

\section{Results and Discussion}

\section{Colonisation}

Among the total isolates, 19 cases (17.59\%) were classified as those causing infection (BSI+HCAP), $89 \quad(82.41 \%) \quad$ being characterised as colonisers. Colonisation rates due to Acinetobacter species were significantly higher than Infections ( $\mathrm{p}$-value 0.001) ( $\mathrm{p} \leq 0.05)($ Table 1).

The mean age of presentation in this study was 51.64 years with a median of 58 years, standard deviation of 22.95 years and standard error of mean of 2.21. The range was $0-89$ years. (Table 2)

There were 93 males $(86.11 \%)$ and 15 females $(13.88 \%)$. Percentage of males who developed clinically significant association with Acineobacter species was $73.68 \%$ (14) and percentage of females who developed such association was $26.3 \%$ (5). Fisher's exact test was used in this test (Table 3 ). 
Gender was not a statistically significant factor for the development of infection due to Acinetobacter species. p-value was 0.1355 . This was considered not significant.

\section{Health Care Associated Pneumonia}

Eighty six consecutive specimens with Acinetobacter species isolated in various respiratory specimens were obtained over the study period.

\section{Colonization}

Based on CDC criteria , 14 cases (16.27\%) of HCAP and 72 cases $(83.72 \%)$ of colonisation due to Acinetobacter species were identified. Colonisation rates due to Acinetobacter species were significantly higher than HCAP. P-value was calculated using binomial test which was 0.000 showing a statistical difference between the two groups. Patients who developed HCAP within 96 hours of admission in ICU were categorised as having "early-onset HCAP" and those who developed after 96 hours were classified as "late-onset HCAP." Out of 14 patients $4(28.57 \%)$ developed "early HCAP" while $10(71.42 \%)$ were of "late HCAP" group where Acinetobacter species was the causative aetiology.p-value was calculated using Fisher's exact test.

\section{Demography}

The mean age of patients in this cohort was 55 years. The mean age of presentation of HCAP in this age group was 46.39 years with a median of 50.50 years, standard deviation of 30.80 and standard error of mean of 8.23 . The range was 1 to 85 years. while mean age of presentation of non HCAP group was 56.61 years, median of 61 years, standard deviation of 18.77 and standard error of mean of 2.23 . Range was 3 to 89 years.
Most of the patients in the study were males $(\mathrm{n}=76,88.37 \%)$ while the number of females was 10 (11.62\%). Twelve males $(85.71 \%)$ presented with HCAP and two females presented with the same (14.28\%).P-value was 0.6635 which was not statistically significant.

\section{Risk Factors}

Among the risk factors, tracheostomy was found to be statistically significant.(p-value $<$ $0.05)$ In our study, $7(50.00 \%)$ of the patients with HCAP had tracheostomy while 14 non HCAP patients(19.44\%) had tracheostomy. (Table 6)

\section{Orotracheal vs Nasotracheal Intubation}

Orotracheal intubation was done on 62 patients $(72.09 \%)$. Three patients had nasotracheal intubation (3.4\%), Six $(9.67 \%)$ patients with orotracheal ventilation developed HCAP while 1 patient (33.33\%) with nasotracheal intubation developed HCAP. Routes of intubation were not statistically significant risk factors for the development of HCAP.

\section{Mode of Feeding}

All the HCAP i.e.14 patients had ryle's tube (enteral) as a mode of feeding (100\%). 70 $(97.22 \%)$ non HCAP patients also had ryle's tube as the mode of feeding.

No significant statistical association of RT feed or parenteral mode was seen with the development of HCAP due to Acinetobacter species.

\section{Average Length of ICU Stay}

The average length of stay in Hospital for patients who developed HCAP with Acinetobacter species was 27.28 days 
whereas the mean for Non-HCAP patients was appreciably low at 19.30 days. Student-t test was used for analysis.

However there was no statistical difference when ICU stay was compared in both the groups.

\section{Blood Stream Infections}

Based on CDC criteria, in this study BSI was diagnosed in only 5 patients $(22.72 \%)$ colonisation by Acinetobacter species was found in a total of 17 cases $(77.27 \%)$

Colonisation rates were significantly higher than BSI. (P-value=0.0169).

\section{Demography}

There were 17 males $(77.27 \%)$ and there were 5 females $(22.72 \%)$ in this study. Two males (40\%) and three females (60\%) developed blood stream infection during this period. One out of two males was neonate. P-value was calculated Fisher's exact test was 0.1358 which was considered not significant.

Mean age of patients presenting with BSI was 40.80 and median age was 42 years with standard deviation of 28.12 and standard error of mean 12.58. The range was from 0-75 years. Mean age of presentation of non-BSI patient was 40.65 years, range between 0-78 years and median age was 37 Years with standard deviation of 26.13 and standard error of mean 6.34. Age was not considered statistically significant (student $\mathrm{t}$ test $\mathrm{p}$-value $=0.8333$ )

\section{Risk Factors}

Among the risk factors considered above in the development of Blood stream infections, Immune suppression had a statistically significant value $(\mathrm{p}$-value $\leq 0.05)$ (Table 4$)$.

\section{Catheter Characteristics}

All central line catheter used were made up of polyurethane material and they were non antibiotic coated and non-tunnelled. All i.e. 5 patients who developed BSI had a central access (100\%), while among the colonisers central line access was present in 15 patients (88.23\%). P-value by Fisher's exact test was 1 .

\section{Route of Insertion}

Out of 22 patients, 16 patients were on internal jugular access, hence incidence of BSI in this group was $25 \%(4), 3$ patients were on femoral access, incidence of BSI was $33.33 \%(1), 1$ patient was on subclavian access, incidence of BSI in this group was $0 \%$, PICC was present in 1 patient and incidence was $100 \%(1)$ incidence was highest with PICC followed by femoral access. Subclavian access was found to be showing the least incidence of BSI .p-value was calculated fisher's exact test.

Various Routes of insertion of central catheters were not statistically significant for the development of BSI due to Acinetobacter species (Table 5).

\section{Type of Catheter}

Multi-lumen and single lumen catheters.Incidence of BSI was $80 \%$ (4) in multilumen catheters. In single lumen catheters it was $20 \%$ (1). Therefore incidence was higher in multilumen central catheters. Out of 22 patients followed 18 had multilumen central catheters and 3 were with single lumen catheters. P-value calculated using Fisher Exact Test However it was not statistically significant.

Multiple Catheters (more than one access line catheters) 
Out of 22 patients multiple catheters were present in 8 patients. Four patients developed BSI with multiple catheters (50\%) compared to $1(7.7 \%)$ patient with absence of multiple catheters who developed BSI. P-value was calculated using Fishers Exact test. There was a statistical difference between the presence and absence of multiple catheters $(\mathrm{p}<0.05)$

\section{Mode of Feeding}

All the BSI patients had ryle's tube (enteral) as a mode of feedoing (100\%) and all non BSI patients also had ryle's tube as the mode of feeding.

No significant statistical association of RT feed or parenteral mode was seen with the development of BSI due to Acinetobacter species.

\section{Average of Length of ICU Stay}

The average length of stay of cases admitted in the ICU ' $\mathrm{S}$, who developed blood stream infection was 25 days. When compared this with colonisers, it was higher than the colonisers in whom the average length of stay was only 15 days. Students t-test was used in this study.

However p-value was not statistically significant when average length of ICU stay was considered.

\section{Species Isolated}

Twenty one isolates were identified as $A$. baumannii complex and one isolate in this group was identified as A. lwoffi. Acinetobacter baumannii complex was responsible for all 5 cases of blood stream infections in this study (100\%).

No statistical significance was seen when species of Acinetobacter were considered for the development of BSI.
Acinetobacter species has emerged an important nosocomial pathogen that is often multidrug resistant and associated with lifethreatening infections (Bergogue-Berezin and Towner, 1996). In our Hospital, the overall colonisation rate vs the infection due to Acinetobacter species rate was calculated to be $82.41 \%$ and $17.59 \%$ (HCAP+BSI) and was found to be significantly in favour of colonisation indicating that they colonise more often than actually causing infection.(pvalue0.001). Therefore, mere isolation of Acinetobacter species should not be considered as significant and needs to be always clinically correlated. Accordingly a judicious use of antimicrobials for the same in the clinical settings is warranted, and should bee done after confirmation of infection.

Acinetobacter baumannii calcoaceticus complex is most frequently isolated species among Acinetobacter species in the ICU settings. Among the Acinetobacter species, it is also most commonly associated with the infections in the ICU'S. In our study, Acinetobacter species other than A.baumanniii- calcoaceticus complex were not associated with any infections.

Tracheostomy was a significant risk factor associated with the development of HCAP due to Acinetobacter species. Immunosuppression and presence of multiple catheters are independent risk factors in blood stream infections.In our study Immunosuppression was found to be significantly higher in the BSI group compared to the Non-BSI group. Likewise Presence of multiple catheters was also associated with significantly higher risk of BSI due to Acinetobacter species and emerged as another risk factor for its development. 
Table.1 Infection Vs Colonisers due to Acinetobacter Species

\begin{tabular}{|l|l|l|}
\hline Category & No. of cases $\mathrm{n}=108 \mathrm{~N} \%$ & $\mathrm{p}$-value \\
\hline Infection (BSI+HCAP) & $19(17.59)$ & 0.001 \\
\hline Colonisers & $89(82.41)$ & \\
\hline
\end{tabular}

Table.2 Age Characteristics of the Study Population

\begin{tabular}{|l|l|l|l|l|}
\hline \multicolumn{6}{|l|}{ Age characteristics (study population) $\mathrm{n}=108 \mathrm{~N} \%$} \\
\hline Mean(yrs) & Median(yrs) & Range(Yrs) & $\begin{array}{l}\text { Standard } \\
\text { deviation }\end{array}$ & $\begin{array}{l}\text { Standard error of } \\
\text { mean }\end{array}$ \\
\hline 51.64 & 58 & $0-89$ & 22.95 & 2.21 \\
\hline
\end{tabular}

Table.3 Gender Characteristics of the Study Population

\begin{tabular}{|l|l|l|l|}
\hline Gender & Infection $\mathrm{n}=19(\mathrm{~N} \%)$ & Colonizer $\mathrm{n}=89 \mathrm{~N} \%$ & $\mathrm{p}$-value \\
Males & $14(73.68)$ & $79(88.76)$ & 0.1355 \\
\hline Females & $5(26.3)$ & $10(11.23)$ & \\
\hline
\end{tabular}

Table.4 Univariate Analysis of Risk Factors Associated with Blood Stream Infections

\begin{tabular}{|l|l|l|l|}
\hline VARIABLE & $\begin{array}{c}\text { BSI Group } \\
\mathrm{n}=5(\mathrm{~N} \%)\end{array}$ & $\begin{array}{c}\text { Non BSI Group } \\
\text { (colonizers) } \mathrm{n}=17(\mathrm{~N} \%)\end{array}$ & P-value \\
\hline Hypertension & $2(40)$ & $3(17.64)$ & 0.548 \\
\hline COPD & $0(0)$ & $0(0)$ & 1 \\
\hline CAD & $0(0)$ & $2(11.11)$ & 1 \\
\hline CRF & $0(0)$ & $2(11.11)$ & 1 \\
\hline CVA & $0(0)$ & $2(11.11)$ & 1 \\
\hline Diabetes & $2(40)$ & $4(23.52)$ & 1 \\
\hline Immuno-suppression & $2(40)$ & $0(0)$ & $\mathbf{0 . 0 4 3 3}$ \\
\hline Chronic lung disease & $0(0)$ & $0(0)$ & 1 \\
\hline Chronic liver disease & $0(0)$ & $2(11.11)$ & 1 \\
\hline Steroids & $0(0 \%)$ & $0(0)$ & 1 \\
\hline Immuno-suppressives & $0(0 \%)$ & $1(5.88)$ & 1 \\
\hline Previous antibiotics & $5(100 \%)$ & $16(94.11)$ & 1 \\
\hline
\end{tabular}


Table.5 Univariate Analysis of Risk Factors Associated with Catheter Characteristics

\begin{tabular}{|l|l|l|l|}
\hline \multicolumn{1}{|c|}{ VARIABLE } & \multicolumn{1}{|c|}{$\begin{array}{c}\text { BSI group } \\
\mathrm{n}=5(\mathrm{~N} \%)\end{array}$} & \multicolumn{1}{|c|}{$\begin{array}{c}\text { Non BSI group } \\
\mathrm{n}=17(\mathrm{~N} \%) \text { (Colonisers) }\end{array}$} & \multicolumn{1}{|c|}{ P-value } \\
\hline Route of insertion & & & 1 \\
\hline Internal jugular & $4(80)$ & $1(5.8)$ & 1 \\
\hline Subclavian & $0(0)$ & $2(11.76)$ & 1 \\
\hline Umblical & $0(0)$ & $2(11.76)$ & 1 \\
\hline Femoral & $1(20)$ & $0(0)$ & 0.227 \\
\hline PICC & $1(100)$ & & 1 \\
\hline Type of catheter & & $14(77.77)$ & 1 \\
\hline Multilumen & $4(80)$ & $2(11.76)$ & 1 \\
\hline Single lumen & $1(20)$ & & \\
\hline
\end{tabular}

Table.6 Univariate analysis of risk factors associated with Health care associated pneumonia

\begin{tabular}{|c|c|c|c|}
\hline Variable & $\begin{array}{l}\text { HAP Group } \\
n=14(N \%)\end{array}$ & $\begin{array}{l}\text { Non HAP Group } \\
\text { (Colonizers) } \\
\mathrm{n}=72(\mathrm{~N} \%)\end{array}$ & $\mathrm{p}$-value \\
\hline Hypertension & $4(28.57)$ & $37(51.38)$ & 0.146 \\
\hline COPD & $1(7.14)$ & $15(20.83)$ & 0.452 \\
\hline CAD & $1(7.14)$ & $15(20.83)$ & 0.452 \\
\hline $\mathrm{CRF}$ & $3(21.42)$ & $9(12.50)$ & 0.404 \\
\hline CVA & $2(14.28)$ & $6(8.33)$ & 0.329 \\
\hline Diabetes & $3(21.42)$ & $19(26.38)$ & 1 \\
\hline Immuno-suppression & $4(28.57)$ & $19(26.83)$ & 1 \\
\hline Chronic lung disease & $0(0)$ & $1(1.38)$ & 1 \\
\hline Chronic liver disease & $1(7.14)$ & $4(5.55)$ & 1 \\
\hline Steroids & $2(14.28)$ & $3(4.16)$ & 0.185 \\
\hline Immuno-suppressives & $0(0)$ & $2(2.77)$ & 0.738 \\
\hline Previous antibiotics & $10(71.42)$ & $55(76.38)$ & 0.738 \\
\hline Tracheostomy & $7(50)$ & $14(19.44)$ & 0.0356 \\
\hline
\end{tabular}


Figure.1 Comparison of Percentage of Infection and Colonisers Due to Acinetbacter Species

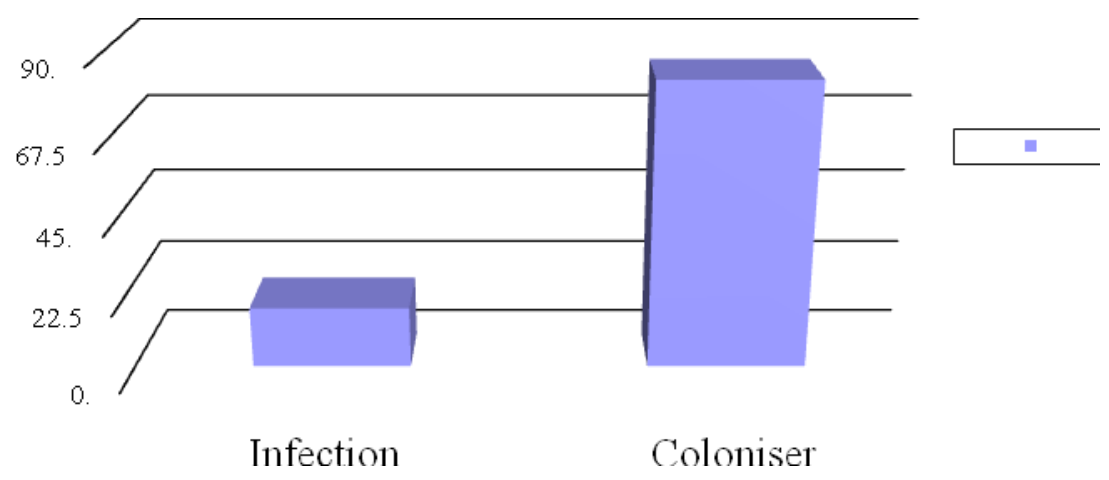

Also Potential risk factors for Acinetobacter infection in many studies are prior use of broad-spectrum antibiotics (Bouvet and Grimont, 1987). However no such correlation was found in our study.

The average length of ICU stay in the BSI group was higher than colonisers (25 Vs. 15.25 days) and though not clinically significant, yet was similar to other studies. The average length of stay in Hospital for patients who developed HCAP with Acinetobacter species was 27.28 days whereas the mean for Non-HCAP patients was appreciably low at 19.30 days.

HCAP and BSI bundle as customised for local conditions prevailing in a particular hospital has been shown to be an effective tool in decreasing the likelihood of HCAP when followed rigorously. Similarly preventive measures for BSI decrease the likelihood of BSI.

In conclusion, this prospective study was conducted to ascertain the role of Acinetobacter as a Pathogen in Healthcare Associated Pneumonia (HCAP) and Blood stream Infections(BSI). Colonisation rate vs the infection $(82.41 \%$ vs $17.59 \%)$ due to Acinetobacter species indicated Acinetobacter are mole likely to be colonisers in the ICU settings.
Tracheostomy was a significant risk factor associated with the development of HCAP. Immunosuppression and presence of multiple catheters was found to be significantly higher in the BSI group compared to the Non-BSI group. This study highlights the fact that mere isolation of Acinetobacter species in blood and respiratory specimens should not be always considered as significant and needs to be always clinically correlated to establish its pathogenicity. Accordingly a judicious use of antimicrobials for the same in the clinical settings is warranted, and should bee done after confirmation of infection.

\section{Acknowledgement}

Dr. Kalyan Mukherjee (Department of Surgery) Indraprastha Apollo Hospitals.

\section{References}

Koenig, S.M., Truwit, J.D. 2006. VentilatorAssociated Pnuemonia: Diagnosis, Treatment and Prevention. Clin. Microbiol. Rev., 637-657.

Collee, G.J., Fraser, A.G., Marimon, B.P., Simmons, A. 2006. Editors. Mackie and Mc Cartney. Prac. Med. Microbiol., 14th ed. 294: 2006.

Nosocomial Infections Due To Acinetobacter Species. 2006. Clinical 
Findings, Risk And Prognostic Factors Nosocomial Iinfections Due To Acinetobacter Species: Clinical findings, risk and prognostic factors. Indian J. Med. Microbiol., 24(1): 3944.

Dijkshoorn, L., Aucken, H., Gerner-Smidt, P., Janssen, P., Kaufmann, M.E., Garaizar, J., Ursing, J., Pitt, T.L. 1996. Comparison of outbreak and nonoutbreak Acinetobacter baumannii strains by genotypic and phenotypic methods. J. Clin. Microbiol. 34: 1519-1525.

Heinemann, B., Wisplinghoff, H., Edmond, M., Seifer, H. 2000. Comparative activities of ciprofloxacin, clinafloxacin, gatifloxacin, gemifloxacin, levofloxacin, moxifloxacin, and trovafloxacin against epidemiologically defined Acinetobacter baumannii strains. Antimicrob. Agents Chemother., 44: 2211-2213.

Jawad, A., Seifert, H., Snelling, A.M., Heritage, J., Hawkey, P.M. 1998. Survival of Acinetobacter baumannii on dry surfaces: comparison of outbreak and sporadic isolates. J. Clin. Microbiol., 36: 1938-1941.

Koeleman, J.G., Van der Bijl, M.W.J., Stoof, C.M., Vandenbroucke-Grauls, Savelkoul, P.H. 2001. Antibiotic resistance is a major risk factor for epidemic behavior of Acinetobacter baumannii. Infect. Control Hosp. Epidemiol., 22: 284-288.

Teresa, C., Horan, M.P.H., Mary Andrus, R.N., BA, C.I.C., Margaret, A., Dudeck. CDC/NHSN surveillance definition of health care-associated infection and criteria for specific types of infections in the acute care setting. AJIC.

Central line associated blood stream infections(CLABSI)http://www.NHS N_training_CLABSI revised_ april_ 08_slides.pdf

Bloodstream Infection Event (Central LineAssociated Bloodstream Infection and Non-central line-associated Bloodstream Infection. www.cdc.gov/nhsn/PDFs/pscManual/ 4PSC_CLABScurrent.pdf

Pneumonia (Ventilator-associated [VAP] and non-ventilator-associated Pneumonia [PNEU]) Event. http://www.cdc.gov/nhsn/PDFs/pscM anual/6pscVAPcurrent.pdf

Bergogue-Berezin, E., Towner, K.J. 1996. Acinetobacter species as nosocomial pathogen: Microbiological, clinical and epidemiological features. Clin. Microbiol. Rev., 9: 148-65.

Bouvet, P.J.M., Grimont, P.A.D. 1987. Identification and biotyping of clinical isolates of Acinetobacter. Ann. Institut. Pasteur. Microbiol., 138: 569-78.

\section{How to cite this article:}

Ruchi Girotra, Raman Sardana, Reetika Dawar, Leena Mehndiratta,

Sudha

Kansal, Rajesh Chawla Ruby Naz and Narendra Agarwal. 2016. Role of Acinetobacter as pathogen of Health care Associated Pneumonia and Blood Stream Infections in ICU Setting in a Tertiary Care Hospital in New Delhi. Int.J.Curr.Microbiol.App.Sci. 5(3): 502-512. doi: http://dx.doi.org/10.20546/ijcmas.2016.503.059 